\title{
AVALIAÇÃO DOS SERVIÇOS DE SAÚDE: UMA PESQUISA COM USUÁRIOS DE UNIDADES BÁSICAS VINCULADAS AO PROGRAMA PRÓ-PET SAÚDE
}

\author{
EVALUATION OF HEALTH SERVICES: A RESEARCH WITH USERS OF BASIC UNITS LINKED \\ TO THE PRÓ-PET HEALTH PROGRAM
}

Ana Claudia da Rosa', Gabriela Rorato Guarienti ${ }^{2}$, Matheus Frohlich Marquetto ${ }^{3}$, Juliano Pereira Régis Garcia ${ }^{4}$, Ana Luiza Rossato Facco ${ }^{5}$, Laís Vieira Trevisan ${ }^{6}$, Ulisses Arthur Carboni ${ }^{7}$, Jaqueline Selinske ${ }^{8}$, Amanda Medeiros ${ }^{9}$, Pâmela Rodrigues ${ }^{10}$, Marcia Zampieri Grohmann ${ }^{11}$, Luciana Flores Battistella ${ }^{12}$, Marli Matiko Campos ${ }^{13}$

RECEBIDO EM: 03/08/2017 / APROVADO EM: 15/10/2017

DOI: $10.5902 / 2317175826077$

\section{RESUMO}

Este trabalho tem por objetivo colaborar para o entendimento do fenômeno das migrações humanas, bem como das redes e das etapas dos processos de acolhimento de imigrantes na cidade de São Paulo, analisando brevemente o papel desempenhado por organizações da sociedade civil. Para isso, pesquisaram-se quais são as características atuais do fenômeno no território brasileiro. Por fim, realizou-se um trabalho de campo em que foram entrevistados 14 estrangeiros, sendo nove imigrantes e cinco refugiados, a partir do qual foi possível traçar algumas redes de apoio e identificar maneiras pelas quais são construídas relações com indivíduos, famílias e instituições. Concluímos que as organizações da sociedade civil desempenham um importante papel na socialização e assistência tanto do refugiado em situação de vulnerabilidade social quanto do imigrante de altos estratos socioeconômicos, na sua fixação na cidade de São Paulo. Outros fatores relevantes identificados foram: os laços familiares, a condição socioeconômica prévia à chegada e as redes de compatriotas, oportunidades nas quais os sujeitos podem recriar suas identidades sociais e os sentimentos de pertença, conforme adesão da análise discursiva proposta por Fairclough.

Palavras-chave: imigração; territórios identitários, redes de pertencimento, São Paulo, deslocamento.

\footnotetext{
1 Mestranda em Administração - PPGAVUFSM email- ana.claudiadarosaa@gmail.com 2 Mestranda em Design - UFRGS email- gabiguarienti@gmail.com

3 Doutorando em Administração - PPGA - UFSM email- matheusmarquetto@yahoo.com.br

4 Mestrando em Administração - PPGA - UFSM email- julianogarciamg@gmail.com

5 Mestre em Administração - PPGA - UFSM - Professora Substituta da UFSM email- analuiza.rfacco@gmail.com

6 Graduanda em Administração 8o semestre - UFSM email- laisvtrevisan@gmail.com

7 Graduado em Administração - UFSM email- uacarboni@gmail.com

8 Mestre em Administração - PPGA - UFSM email- jaquelinesilinske@gmail.com

9 Graduanda em Administração - UFSM email- amanda.m1713@yahoo.com.br

10 Graduanda em Administração - UFSM email- ana.claudiadarosaa@gmail.com

11 Professora da UFSM email-marciazg@gmail.com

12 Professora da UFSM email- luttibattistella@gmail.com

13 Professora da UFSM email- marlimatiko@yahoo.com.br
} 
AVALIAÇ̃̃O DOS SERVIÇOS DE SAÚDE:

UMA PESQUISA COM USUÁRIOS DE UNIDADES BÁSICAS VINCULADAS AO PROGRAMA PRÓ-PET SAÚDE

\begin{abstract}
The present research aimed to measure the quality and satisfaction of the services rendered and to verify how the profile of the users interferes in the evaluation of these dimensions. For this, a quantitative and descriptive study was conducted with 373 users of ten Basic Health Units (UBS) linked to PRÓ-PET-Saúde in the city of Santa Maria / RS. The satisfaction scale was used by Oliver (1997) to measure the quality of the SERVQUAL Scale proposed by Parasuraman, Zeitham/ and Berry (1998). In the analysis of the means and standard deviations between each UBS researched, it was identified that, in general, the UBS were well evaluated. Regarding the impact of the profile of the users, it was verified that this profile generated a significant impact in the evaluation on the services provided by the UBS. Thus, the results found may serve as a basis for the constant improvement of the public health services offered

by the health-care providers at PRÓ-PET-Saúde in Santa Maria / RS, as it revealed users' assessment of various aspects of care.
\end{abstract}

Keywords: Sistema Único de Saúde (SUS); PRÓ-PET-Saúde; Satisfaction; SERVQUAL.

\title{
1 Introdução
}

Os Ministérios da Saúde e da Educação vem propondo projetos que incitam a parceria entre universidades e serviços de saúde, como o Programa Nacional de Reorientação da Formação em Saúde (PRÓ-Saúde) e o Programa de Educação pelo Trabalho para a Saúde (PET-Saúde) (PEREIRA, 2007). Tais programas constituem o PRÓ-PET-Saúde, que compõe ações direcionadas para o fortalecimento da atenção básica e da vigilância em saúde, de acordo com princípios do Sistema Único de Saúde (SUS).

Segundo Ferreira et al. (2012), o Programa PRÓ-PET-Saúde tem como pressuposto a educação pelo trabalho baseada na integração entre ensino, serviço e comunidade. Paralela à busca por essa integração, a inserção dos próprios usuários na avaliação dos serviços de saúde tem sido muito valorizada, por estar potencialmente relacionada a uma maior adequação no uso destes, além de articular os aspectos econômicos de eficiência e eficácia dos serviços prestados (ESPERIDIÃO e TRAD, 2005).

Assim, a avaliação dos usuários pode produzir informação para a melhoria das intervenções em saúde (VIEIRA-DA-SILVA, 2005). Com isso,essa avaliação necessita se tornar um procedimento contínuo na gestão destes serviços, cujos elementos devem manter articulação entre as ações técnicas e administrativas (RIBEIRO, 2010). Considerando essa perspectiva, o objetivo do processo de avaliação é a busca pela maior eficiência e eficácia dos serviços, e isso só poderá ser obtido se a avaliação levar em conta a opinião e as necessidades dos usuários dos serviços de saúde (TANAKA e MELO, 2004).

Portanto, para possibilitar o contínuo aprimoramento dos serviços de saúde oferecidos à população, se torna importante promover a constante avaliação destes sob a perspectiva dos usuários que são atendidos pelo programa PRÓ-PET-Saúde. A presente pesquisa objetivou mensurar a qualidade e a 
ANA CLAUDIA DA ROSA, GABRIELA RORATO GUARIENTI, MATHEUS FROHLICH MARQUETTO, JULIANO PEREIRA RÉGIS GARCIA, ANA LUIZA ROSSATO FACCO, LAÍS VIEIRA TREVISAN, ULISSES ARTHUR CARBONI, JAQUELINE SELINSKE, AMANDA MEDEIROS, PÂMELA RODRIGUES, MARCIA ZAMPIERI GROHMANN, LUCIANA FLORES BATTISTELLA, MARLI MATIKO CAMPOS

satisfação dos serviços prestados e verificar como o perfil dos usuários interfere na avaliação dessas dimensões. Assim, a qualidade foi mensurada pela adaptação da Escala SERVQUAL proposta por Parasuraman, Zeithaml e Berry (1998), através das dimensões confiabilidade, empatia, presteza, segurança e tangibilidade, e a dimensão satisfação foi medida pela Escala de Satisfação de Oliver (1997).

Para atender aos objetivos propostos, realizou-se uma pesquisa quantitativa e descritiva, com a aplicação de questionários junto a 373 usuários dessas prestabilidades de saúde de dez Unidades Básicas de Atendimento (UBS) vinculadas ao programa PRÓ-PET-Saúde na cidade de Santa Maria/RS.

\section{PRÓ-PET-Saúde}

No ano de 2007, a Secretaria de Gestão do Trabalho e da Educação na Saúde (SGTES) criou o PET-saúde, elaborado como uma oportunidade de elo entre o ensino-serviço-comunidade, objetivando formar grupos de ensino -aprendizagem conduzidos por tutores e destinados em áreas consideradas importantes para o SUS, servindo, assim, como mecanismo para a proximidade das políticas públicas e de saúde e a prática dos serviços (CEZARIO et al., 2015).

O PET-Saúde é definido como uma estratégia do PRÓ-Saúde para favorecer a formação de grupos de aprendizagem tutorial em áreas prioritárias para o SUS, adicionando os acadêmicos das graduações da área da saúde nos espaços dos serviços. O programa mostra uma prática de interdisciplinaridade que patrocina o aprendizado de um novo conhecimento para a confecção de outras práticas do saber de acordo com as necessidades das pessoas. Desse modo, o PET-Saúde pode ser caracterizado como uma tática dinâmica capaz de auxiliar para um melhor vínculo entre a vida acadêmica e os serviços de saúde, proporcionando melhor reflexão para o aperfeiçoamento de um bom profissional que atenda os princípios do SUS, de acordo com a necessidade da população (ARAÚJO et al., 2012).

Cabe destacar que no ano de 2012 ocorreu a união do PET-Saúde com o PRÓ-Saúde, resultando no PRÓ-PET-Saúde, que vem desenvolvendo ações de promoção, prevenção, controle social e pesquisa nos serviços de saúde, com intuito de promover a integração ensino-serviço e tornou possível o contato com a realidade do SUS (SILVA, SCAPIN e BATISTA, 2011). Assim, a importância do PRÓ-PET-Saúde ocorre pelo fato de que as experiências vivenciadas no projeto oportunizam estabelecer um novo olhar para as várias possibilidades que a formação em saúde constitui para o fortalecimento do SUS.

A partir do exposto, insere-se o contexto do SUS, criado em 1988 pela Constituição Federal Brasileira, configurando-se como um dos maiores sistemas políticos de saúde no mundo, garantindo o acesso integral, universal e gratuito aos mais de 180 milhões de brasileiros (MINISTÉRIO DA SAÚDE, 2017). Desse modo, para a garantia de acesso a atenção de saúde foi criada a UBS, 
AVALIAÇ̃̃O DOS SERVIÇOS DE SAÚDE:

como principal porta de entrada a centros de comunicação com toda a rede de atenção à saúde, sendo instalada perto de onde as pessoas moram, trabaIham, estudam e vivem, objetivando desenvolver uma atenção integral que impacte na situação da saúde e autonomia das pessoas. Nas UBS é possível receber atendimentos básicos e gratuitos em Pediatria, Ginecologia, Clínica Geral, Enfermagem e Odontologia, integrando como principais serviços às consultas médicas, inalações, injeções, vacinas, exames laboratoriais e fornecimento de medicação básica, além de encaminhamentos para especialidades (MINISTÉRIO DO PLANEJAMENTO, 2017).

Sendo assim, é possível observar o desempenho do PRÓ-PET-Saúde como incentivo de diversas atividades que valorizam o aprendizado e atualização do dia-a-dia das práticas em atenção básica. O PRÓ-PET-Saúde possibilita a elaboração comum de várias ideias e ações, já que inclui a participação do instrumento docente, do serviço e da comunidade. Portanto, o PRÓ-PET-Saúde tem atuado como importante ferramenta para formação de profissionais habilitados a praticar atividades de acordo com a procura do SUS, promovendo qualidade de vida à população (ARAÚJO et al., 2012).

As práticas executadas pelos grupos PRÓ-PET-Saúde têm chegado à interação entre ensino e serviço, ajudando efetivamente na instrução acadêmica, no espaço de atenção à saúde e, principalmente, na sociedade, originando novos olhares para as necessidades de saúde dos indivíduos. Assim, impulsionam a responsabilidade e o compromisso dos diferentes atores, componentes do programa, edificando potencialidades para encarar limitações, construindo capacidades e soluções cabíveis para a melhoria na qualidade dos serviços de saúde (MORAIS, et al., 2012).

\section{Avaliação dos serviços de saúde}

A avaliação de um programa de saúde é necessária para obter conhecimento sobre a realidade da situação encontrada e se os objetivos iniciais foram alcançados, além de servir como base para a reorientação de mudanças no serviço. Desse modo, muitos desafios são encontrados, por ser uma área complexa e receber influência de vários fatores, o que dificulta a determinação dos objetivos a serem avaliados (MEDINA et al., 2005).

Segundo Medina et al. (2005) o objetivo de avaliar a qualidade é para determinar o nível de resultado das profissões na área da saúde, para assim evitar o abuso ou a incompetência. Por outro lado, os autores afirmam que o monitoramento da qualidade é para praticar a supervisão constante, para que, quando surgirem irregularidades, as mesmas possam ser sanadas o mais rápido possível, sendo que essa avaliação tem duas dimensões: a primeira referente ao desempenho técnico, que se caracteriza pelo profissional ter conhecimento pelo serviço e tecnologia médica, para o bem-estar do paciente. A segunda se determina pela relação pessoal do paciente, respeitando valores 
ANA CLAUDIA DA ROSA, GABRIELA RORATO GUARIENTI, MATHEUS FROHLICH MARQUETTO, JULIANO PEREIRA RÉGIS GARCIA, ANA LUIZA ROSSATO FACCO, LAÍS VIEIRA TREVISAN, ULISSES ARTHUR CARBONI, JAQUELINE SELINSKE, AMANDA MEDEIROS, PÂMELA RODRIGUES, MARCIA ZAMPIERI GROHMANN, LUCIANA FLORES BATTISTELLA, MARLI MATIKO CAMPOS

éticos, desejos e necessidades dos pacientes.

No Brasil, o objetivo de avaliar o SUS tem como base oferecer apoio às decisões que serão tomadas, reconhecer as anormalidades e dirigir os serviços e práticas a serem desenvolvidas, além de verificar o impacto do serviço de saúde sobre a população (BRASIL, 2005). Vale lembrar que, na saúde, a avaliação tem como objetivo melhorar as ações de atenção e gerar processos apropriados com os princípios do SUS (BRASIL, 2005).

De acordo com o Ministério da Saúde, a institucionalização da avaliação necessita estar ligada à criação de uma política de avaliação no contexto do SUS, na qual a Atenção Básica ( $A B$ ) deve estar incluída nesse processo, agrupando todas as pessoas envolvidas (usuários, gestores, profissionais da saúde, pesquisadores) (BRASIL, 2005). Assim, a avaliação na área da saúde não deve ser apenas o monitoramento das práticas e regras, mas também uma maneira democrática de dar poder aos usuários na confirmação de seus direitos, pois, as pessoas caracterizam-se como um dos atores corresponsáveis (AYRES, 2004). Além disso, Gomes et al. (2011) ressaltam que a opinião do indivíduo, além de significar um indicador sensível da qualidade do serviço prestado, é uma categoria considerada estratégica para uma maior adequação dos serviços às suas demandas, fator essencial para a efetivação dos preceitos do SUS.

No Brasil, as pesquisas de opinião e satisfação dos usuários se tornaram mais frequentes a partir de segunda metade da década de 1990, devido ao processo de redemocratização do país e do surgimento da luta pelos direitos sociais e, especificamente, do acesso às políticas públicas de saúde (ESPERIDIÃO e TRAD, 2005). Assim, no setor público os custos crescentes dos serviços de saúde foram um dos elementos que favoreceram a construção de políticas reformadoras de restrição de gastos e o surgimento de novos modelos de gestão, estimulando assim, o estudo sobre a satisfação do usuário, visando maior transparência, qualidade e eficiência dos serviços (VAITSMAN, 2005).

De acordo com Gouveia (2009), as diversas iniciativas voltadas para a avaliação em saúde vêm sendo desenvolvidas atualmente no Brasil, não se restringindo apenas à academia, mas também permitindo grande multiplicidade de recortes e abordagens metodológicas, devido à sua natureza e abrangência.

Diante disso, um estudo realizado em um município da Região Central do RS, cujo objetivo consistia em avaliar a qualidade da Estratégia Saúde da Família (ESF), verificou uma avaliação negativa da ESF tanto por parte dos usuários, quanto por parte dos profissionais, em que ambos mostraram-se insatisfeitos com a qualidade dos serviços (RIGHI, SCHMIDT e VENTURINI, 2010). A pesquisa de Ribeiro (2010), na cidade de Campina Grande-PB, objetivou avaliar o grau de satisfação de usuários do ambulatório do Hospital Universitário Alcides Carneiro e os resultados apontaram que os usuários do ambulatório encontram-se insatisfeitos com os serviços prestados pela instituição. Ainda, outra pesquisa realizada por Silva (2016), no município de Caraguatatuba, cujo 
AVALIAÇ̃̃O DOS SERVIÇOS DE SAÚDE:

objetivo era identificar a percepção da qualidade e a satisfação dos usuários com relação aos serviços oferecidos pelas UBS, revelou que os usuários também não estão satisfeitos sobre a qualidade de serviço prestada, sendo que as maiores críticas dos usuários foram sobre a falta de confiança e segurança dos funcionários, e também as instalações e equipamentos antiquados.

Diante desse cenário, Pena et al. (2013) afirmam que na área de saúde diversos autores vêm empregando a escala SERVQUAL como um instrumento importante para conhecer as expectativas e necessidades dos usuários, revelando-se como uma escala eficiente para mensurar as percepções e as expectativas sobre a qualidade do serviço. Assim sendo, no presente estudo, a mensuração da opinião dos usuários dos serviços de saúde realiza-se a partir da SERVQUAL de Parasuraman, Zeithaml e Berry (1998).

A literatura contempla que os modelos de qualidade surgiram no final da década de 1970, como resultado de inúmeros trabalhos que objetivavam conceitualizações, operacionalizações e sistematizações para a qualidade dos serviços (PENA et al., 2013). Segundo os autores, avaliar um serviço é mais complexo do que avaliar um produto, pois, enquanto o este é tangível, podendo ser detectado seus defeitos, funcionamento e averiguado sua durabilidade, aquele é comprado, primeiramente, para depois ser produzido e consumido simultaneamente, sendo assim, os serviços são intangíveis, e seu julgamento será feito por meio das experiências de quem os utiliza.

A partir do exposto, o modelo utilizado no estudo é composto por cinco dimensões: tangibilidade, confiabilidade, responsividade ou presteza, garantia ou segurança e empatia (PARASURAMAN, ZEITHAML e BERRY, 1998). O conhecimento da percepção do usuário sob a ótica das cinco dimensões possibilita uma avaliação do grau de satisfação do usuário com a assistência prestada pelas organizações e serviços de saúde (RIBEIRO, 2010).

A dimensão tangibilidade diz respeito à aparência física das instalações, equipamentos, quadro de trabalhadores e material percebidos pelos sentidos humanos (PENA et al., 2013). Ressalta-se que essa dimensão mede questões físicas do local no qual os participantes do PRÓ-PET-Saúde, conhecidos como petianos, prestam serviços à comunidade e que seu resultado não depende somente do investimento do PRÓ-PET-Saúde, mas reflete o investimento realizado por órgãos executivos, como Prefeitura e Secretarias.

Conforme Pena et al. (2013), a confiabilidade refere-se a habilidade do funcionário em executar de forma segura e eficiente o serviço, e retrata um desempenho consistente. Para os mesmos autores, o fator responsividade ou presteza remete à disponibilidade do prestador atender voluntariamente aos usuários, prestando um serviço de forma atenciosa e com prontidão. Também de acordo com Pena et al. (2013), o fator garantia ou segurança é identificado como a cortesia, o conhecimento dos trabalhadores e sua habilidade de transmitir confiança. Já a dimensão empatia relata se o prestador de serviço importa-se com o usuário e assiste-o de forma individualizada. 
ANA CLAUDIA DA ROSA, GABRIELA RORATO GUARIENTI, MATHEUS FROHLICH MARQUETTO, JULIANO PEREIRA RÉGIS GARCIA, ANA LUIZA ROSSATO FACCO, LAÍS VIEIRA TREVISAN, ULISSES ARTHUR CARBONI, JAQUELINE SELINSKE, AMANDA MEDEIROS, PÂMELA RODRIGUES, MARCIA ZAMPIERI GROHMANN, LUCIANA FLORES BATTISTELLA, MARLI MATIKO CAMPOS

A satisfação do usuário tornou-se um componente fundamental para medir a qualidade de um serviço (RIBEIRO, 2010). Por isso, diversos pesquisadores passaram a demonstrar preocupação e interesse em conhecer as expectativas, percepções e o sentimento destes acerca da assistência prestada nos serviços de saúde (DONABEDIAN, 1993; LEMME, NORONHA e RESENDE, 1991; RAMOS e LIMA, 2003; TRAVASSOS e MARTINS, 2004; SILVA e FORMIGLI, 1994; WEISS, 1988). Assim, para verificar o grau de satisfação dos usuários da comunidade dos serviços prestados pelas UBS vinculadas ao PRÓ-PET-Saúde, realizou-se uma adaptação da Escala de Satisfação proposta por Oliver (1997). Para o autor, a satisfação pode ser vista "como uma avaliação da surpresa inerente à aquisição de um produto/serviço e/ou à experiência de seu consumo".

\section{Metodologia}

O estudo caracterizou-se como quantitativo e descritivo, utilizando uma adaptação da escala SERVQUAL (PARASURAMAN, ZEITHAML e BERRY, 1988) e da Escala de Satisfação proposta por Oliver (1997) para avaliar o Programa PRÓ-PET-Saúde sob a perspectiva dos usuários dos serviços de saúde.

O cálculo da amostra mínima utilizou como parâmetro a população estimada de Santa Maria/RS em 2015, que era de 276.108 habitantes (IBGE, 2015). Assim, utilizou-se erro amostral de $5 \%$ e parâmetro percentual estimado de 0,8 , e dessa forma a amostra mínima calculada foi de 276 usuários. Portanto, estipulou-se a aplicação de 30 questionários por UBS. A coleta de dados ocorreu juntamente com os usuários durante o horário de funcionamento das UBS. Desse modo, a amostra da pesquisa formou-se por 373 usuários de dez UBS vinculadas ao Programa PRÓ-PET Saúde em Santa Maria/RS, descritas no Quadro 1.

\begin{tabular}{|c|c|c|}
\hline Unidade Básica de Saúde & Amostra mínima calculada & Questionários obtidos \\
\hline Walter Aita & 30 & 36 \\
\hline Alto da Boa Vista & 30 & 35 \\
\hline Erasmo Crosseti & 30 & 34 \\
\hline CAPS Caminho do Sol & 30 & 31 \\
\hline Roberto Binato & 30 & 33 \\
\hline Ambulatório de Saúde Mental & 30 & 35 \\
\hline Wilson Paulo Noal & 30 & 30 \\
\hline São José & 30 & 35 \\
\hline Lídia & 30 & 35 \\
\hline Urlândia & 30 & 34 \\
\hline TOTAL & 330 & 373 \\
\hline
\end{tabular}

Quadro 1 - Cálculos da amostra e questionários obtidos no estudo Fonte: dados da pesquisa

O questionário aplicado era composto por duas partes: 6 perguntas referentes ao perfil dos usuários (sexo, idade, estado civil, escolaridade, renda e Unidade de Saúde (UBS) que o usuário frequenta) e 25 questões sobre os mo- 
AVALIAÇÃO DOS SERVIÇOS DE SAÚDE:

UMA PESQUISA COM USUÁRIOS DE UNIDADES BÁSICAS VINCULADAS AO PROGRAMA PRÓ-PET SAÚDE

delos teóricos, conforme exposto no Quadro 2, sendo que todas as afirmações referentes ao modelo foram operacionalizadas em uma escala Likert de cinco pontos, variando de 1 = "discordo totalmente" a 5 = "concordo totalmente".

\begin{tabular}{|c|c|}
\hline Construto & Variável \\
\hline \multirow{2}{*}{ Confiabilidade } & $\begin{array}{l}\text { C1 - Quando tenho algum problema, a equipe é solidária } \\
\text { C2 - A equipe de profissionais inspira confiança } \\
\text { C3 - A equipe de profissionais efetua o serviço no tempo prometido }\end{array}$ \\
\hline & C4 - A equipe de profissionais mantém meus registros de forma correta \\
\hline Empatia & $\begin{array}{l}\text { Emp1: A equipe de profissionais me dá atenção individual } \\
\text { Emp2: A equipe de profissionais sabe das minhas necessidades } \\
\text { Emp3: A equipe de profissionais tem interesses em minhas necessidades } \\
\text { Emp4: A Unidade de Saúde tem os horários de funcionamento convenientes }\end{array}$ \\
\hline Presteza & $\begin{array}{l}\text { Pre1: A equipe de profissionais informa, exatamente, quando o atendimento acontecerá } \\
\text { Pre2: Eu recebo atendimento imediato da equipe de profissionais } \\
\text { Pre3: A equipe de profissionais está sempre disposta a ajudar as pessoas } \\
\text { Pre4: A equipe de profissionais está sempre preocupada em responder aos pedidos dos usuários }\end{array}$ \\
\hline Segurança & $\begin{array}{l}\text { Seg1: Eu me sinto seguro em ser atendido pela equipe de profissionais dessa Unidade de Saúde } \\
\text { Seg2: A equipe de profissionais é educada comigo } \\
\text { Seg3: A equipe de profissionais tem suporte adequado na Unidade de Saúde para cumprir as } \\
\text { tarefas }\end{array}$ \\
\hline Tangibilidade & $\begin{array}{l}\text { Tan1: A Unidade de Saúde está bem equipada } \\
\text { Tan2: As instalações físicas da Unidade de Saúde são boas } \\
\text { Tan3: A equipe de profissionais na Unidade de Saúde está, adequadamente, vestida e higienizada } \\
\text { Tan4: As instalações físicas da Unidade de Saúde estão conservadas }\end{array}$ \\
\hline Satisfação & $\begin{array}{l}\text { Sat1: Esta é a melhor Unidade de Saúde dentre as que já fui atendido } \\
\text { Sat2: A Unidade de Saúde é exatamente o que eu necessito } \\
\text { Sat3: Esta Unidade de Saúde funciona tão bem como deveria } \\
\text { Sat4: Eu, realmente, gosto desta Unidade de Saúde } \\
\text { Sat5: Eu me sinto mal vindo aqui nesta Unidade de Saúde } \\
\text { Sat6: Estou satisfeito com essa Unidade de Saúde }\end{array}$ \\
\hline
\end{tabular}

Após a coleta, os dados foram tabulados e analisados no Software Statistical Package for the Social Sciences (SPSS) Statics 21. Para a caracterização do perfil dos usuários realizaram-se estatísticas descritivas, na sequência o cálculo do Alpha de Cronbach, a fim de verificar a confiabilidade dos construtos, após calculou-se as médias e desvios-padrões para avaliar a avaliação da qualidade e satisfação dos serviços de saúde, e por fim, procedeu-se ao Teste T e Teste Anova para verificar como o perfil dos usuários interfere na avaliação dessas dimensões. 
ANA CLAUDIA DA ROSA, GABRIELA RORATO GUARIENTI, MATHEUS FROHLICH MARQUETTO, JULIANO PEREIRA RÉGIS GARCIA, ANA LUIZA ROSSATO FACCO, LAÍS VIEIRA TREVISAN, ULISSES ARTHUR CARBONI, JAQUELINE SELINSKE, AMANDA MEDEIROS, PÂMELA RODRIGUES, MARCIA ZAMPIERI GROHMANN, LUCIANA FLORES BATTISTELLA, MARLI MATIKO CAMPOS

\section{Resultados e discussões}

Os resultados encontram-se divididos em três etapas: (1) Perfil dos usuários das UBS; (2) Avaliação da qualidade e satisfação dos serviços de saúde; (3) Impacto do perfil dos usuários nas avaliações dos serviços das UBS.

\subsection{Perfil dos usuários das UBS}

A amostra de usuários dos serviços de saúde, em relação à distribuição de sexo, formou-se por 250 pessoas do sexo feminino $(67,2 \%)$ e 122 do sexo masculino $(32,8 \%)$.

Em termos de estatísticas descritivas, a idade média dos usuários foi de 45,31 anos, com idade mínima de 18 anos e idade máxima de 84 anos e um desvio-padrão de 16,34 anos, demonstrando heterogeneidade em relação à faixa etária dos usuários de serviços públicos de saúde. A distribuição nas faixas etárias, apresentada na Tabela 1, foi de: 30 usuários até 20 anos (8,1\%), 55 usuários entre 21 a 30 anos (14,8\%), 54 usuários entre 31 a 40 anos (14,6\%), 83 usuários entre 41 a 50 anos (22,4\%), 73 usuários entre 51 a 60 anos (19,6\%), 62 usuários entre 61 a 70 anos (16,6\%) e 14 usuários com mais de 70 anos $(3,8 \%)$. Assim, apesar de uma distribuição homogênea entre as faixas etárias, observou-se uma predominância de usuários na faixa de 41 a 50 anos de idade, com uma representação de $22,4 \%$ do total dos respondentes.

\begin{tabular}{l|c|c} 
Tabela 1 - Faixa etária dos usuários \\
\hline Faixa etária & Frequência & Percentual \\
\hline Até 20 anos & 30 & 08,1 \\
21 a 30 anos & 55 & 14,8 \\
31 a 40 anos & 54 & 14,6 \\
41 a 50 anos & 83 & 22,4 \\
51 a 60 anos & 73 & 19,6 \\
61 a 70 anos & 62 & 16,6 \\
Mais de 70 anos & 14 & 03,8 \\
\hline Total & $\mathbf{3 7 1}$ & $\mathbf{1 0 0 , 0}$ \\
\hline
\end{tabular}

Fonte: dados da pesquisa

Em relação ao estado civil, 132 usuários são solteiros (35,4\%), 179 usuários são casados ou possuem união estável (48\%), 28 usuários são divorciados (7,5\%) e 34 usuários são classificados como outros (9,1\%). Observa-se uma predominância de usuários casados (48\%), representando quase metade da amostra pesquisada.

Quanto à escolaridade dos usuários, na Tabela 2, houve predominância de usuários com ensino fundamental (completo e incompleto) e com ensino médio (completo e incompleto). A distribuição encontrada foi: 21 usuários 
AVALIAÇ̃̃O DOS SERVIÇOS DE SAÚDE:

não frequentaram a escola (5,7\%), 192 usuários possuíam ensino fundamental completo ou incompleto (51,8\%), 128 usuários possuíam ensino médio completo ou incompleto $(34,5 \%)$ e apenas 30 usuários possuíam ensino superior completo ou incompleto (8,1\%). Observa-se que mais da metade da amostra é representada por usuários com ensino fundamental.

Tabela 2 - Escolaridade dos usuários

\begin{tabular}{c|c|c}
\hline Escolaridade & Frequência & Percentual \\
\hline Não frequentou escola & 021 & 05,7 \\
Ensino fundamental completo/incompleto & 192 & 51,8 \\
Ensino médio completo/incompleto & 128 & 34,5 \\
Superior completo/incompleto & 030 & 08,1 \\
\hline Total & $\mathbf{3 7 1}$ & $\mathbf{1 0 0 , 0}$ \\
\hline
\end{tabular}

Fonte: dados da pesquisa

A mensuração da renda dos usuários foi medida por meio do número de salários mínimos recebidos, em média, mensalmente pelo total de membros da família. Destaca-se que 34 usuários $(9,1 \%$ do total) não souberam ou não quiserem responder essa questão.

A distribuição dos usuários, em termos de renda, foi concentrada nas duas faixas mais baixas, conforme demonstra a Tabela 3, demonstrando que quase $75 \%$ da amostra pesquisada ganha até 2 salários mínimos por mês. A renda dos usuários foi de: 148 usuários recebem 1 salário mínimo por mês (43,7\%), 111 usuários recebem 2 salários mínimos por mês (32,7\%), 49 usuários recebem 3 salários mínimos por mês (14,5\%), 20 usuários recebem 4 salários mínimos por mês $(5,9 \%)$ e 11 usuários recebem mais de 4 salários mínimos por mês (3,2\%). Dos onze usuários que formaram o quarto grupo de renda (mais de 4 salários mínimos) um mencionou receber 5 salários mínimos, dois recebem 6 salários mínimos, cinco recebem 7 salários mínimos, dois recebem 9 salários mínimos e um recebe 10 salários mínimos.

Tabela 3 - Renda mensal dos usuários

\begin{tabular}{c|c|c}
\hline Renda mensal & Frequência & Percentual \\
\hline 1 salário mínimo & 148 & 43,7 \\
2 salários mínimos & 111 & 32,7 \\
3 salários mínimos & 049 & 14,5 \\
4 salários mínimos & 020 & 05,9 \\
Mais de 4 salários mínimos & 011 & 03,2 \\
\hline Total & $\mathbf{3 3 9}$ & $\mathbf{1 0 0 , 0}$ \\
\hline
\end{tabular}

Por fim, referente ao número de respondentes por cada unidade de saúde (Tabela 4), verificou-se que 36 pessoas $(9,7 \%)$ são da unidade Walter Aita; as unidades do Alto da Boa Vista, Ambulatório de Saúde Mental, São 
ANA CLAUDIA DA ROSA, GABRIELA RORATO GUARIENTI, MATHEUS FROHLICH MARQUETTO, JULIANO PEREIRA RÉGIS GARCIA, ANA LUIZA ROSSATO FACCO, LAÍS VIEIRA TREVISAN, ULISSES ARTHUR CARBONI, JAQUELINE SELINSKE, AMANDA MEDEIROS, PÂMELA RODRIGUES, MARCIA ZAMPIERI GROHMANN, LUCIANA FLORES BATTISTELLA, MARLI MATIKO CAMPOS

José, Lídia, Maringá possuíram em cada unidade 35 entrevistados $(9,4 \%)$; os postos Erasmo Crosseti e Urlândia tiveram 34 entrevistados (9,1\%); o posto Roberto Binatto foi formado por 33 pessoas (8,8\%); a unidade CAPS Caminho do Sol teve 31 respondentes ( $8,3 \%)$; e o posto Wilson Paulo Noal obteve 30 respondentes $(8,0 \%)$.

Tabela 4 - Unidades de Saúde pesquisadas

\begin{tabular}{c|c|c}
\hline UBS & Frequência & Percentual \\
\hline Walter Aita & 36 & 9,7 \\
Alto da Boa Vista & 35 & 9,4 \\
Erasmo Crosseti & 34 & 9,1 \\
CAPS Caminho do Sol & 31 & 8,3 \\
Roberto Binato & 33 & 8,8 \\
Ambulatório de Saúde Mental & 35 & 9,4 \\
Wilson Paulo Noal & 30 & 8,0 \\
São José & 35 & 9,4 \\
Lídia & 35 & 9,4 \\
Urlândia & 34 & 9,1 \\
Maringá & 35 & $\mathbf{9 , 4}$ \\
\hline Total & $\mathbf{3 7 3}$ & $\mathbf{1 0 0 , 0}$ \\
\hline
\end{tabular}

Fonte: dados da pesquisa

5.2 Avaliação da qualidade e satisfação dos serviços de saúde

A avaliação dos usuários em relação aos serviços recebidos nas UBS de Santa Maria utilizou dois modelos teóricos (PARASURAMAN, ZEITHAML e BERRY, 1988; OLIVER, 1997) dividido em seis dimensões: confiabilidade, empatia, presteza, segurança, tangibilidade e satisfação. Antes de partir para as avaliações das dimensões (médias e desvios-padrões), procedeu-se ao cálculo do Alpha de Cronbach para atestar a confiabilidade das dimensões. Segundo Hair Jr. et al. (2005), o Alpha de Cronbach deve apresentar valor superior a 0,60, porém autores como Pestana e Gageiro (2000) argumentam que em pesquisas de ciências sociais, valores acima de 0,50 são aceitáveis. Verificou-se que todas as dimensões apresentaram confiabilidade satisfatória $(A / p h a \geq 0,5)$, conforme exposto na Tabela 5. Assim sendo, num primeiro momento foram analisadas as médias e desvios-padrões das dimensões, também expostos na Tabela 5, e na sequência, cada dimensão foi trabalhada detalhadamente.

A escala Likert utilizada variou de 1 a 5 , assim sendo, uma média acima do meio da escala é considerada uma boa avaliação (HAIR JR. et al., 2005). Desse modo, a dimensão melhor avaliada pelos usuários foi confiabilidade, demonstrando que os usuários confiam nos profissionais que atendem nos postos de saúde, com média de 4,34 e desvio-padrão de 0,83. A segunda dimensão melhor avaliada foi segurança, revelando que os usuários sentem-se seguros em ser atendidos pelos profissionais de saúde dos postos, com média de 4,22 e desvio-padrão de 0,98. 
AVALIAÇ̃̃O DOS SERVIÇOS DE SAÚDE:

A terceira dimensão melhor avaliada foi empatia, revelando que os entrevistados acreditam que os profissionais de saúde sabem reconhecer as necessidades dos usuários, com média de 4,14 e desvio-padrão de 0,97. A dimensão que obteve quarto lugar foi presteza, que avaliava se os usuários acreditam que a equipe tem conhecimento sobre os serviços prestados, com média de 4,07 e desvio-padrão de 0,84.

Por fim, as dimensões com menores avaliações foram a tangibilidade (média 3,85 e desvio-padrão 1,00) e satisfação (média 3,47 e desvio-padrão $0,87)$, demonstrando que os usuários avaliam menos as instalações e equipamentos das unidades bem como a satisfação pelos serviços prestados.

Analisando-se as médias obtidas foi possível concluir que as UBS pesquisadas foram, de modo geral, bem avaliadas, visto que a escala utilizada variou de 1 (péssimo ou discordo totalmente) a 5 (ótimo ou concordo totalmente) e todas as dimensões apresentaram valores próximos de 4 . Desse modo, os usuários pesquisados sentem confiança e segurança nos serviços prestados pelas UBS atendidas pelo Programa PRÓ-PET-Saúde, afirmam que os profissionais da saúde possuem conhecimento e empatia no atendimento, e atrelam as menores avaliações às condições dos equipamentos e instalações das UBS, bem como à satisfação geral com relação aos serviços prestados.

Em relação aos valores do desvio-padrão, todos foram próximos de 1,00 e, para a escala utilizada, tal valor é considerado pequeno e aponta que não houve grande divergência na avaliação dos serviços de saúde sob a perspectiva dos usuários.

Tabela 5 - Média, desvio-padrão e Alpha de Cronbach das dimensões

\begin{tabular}{c|c|c|c}
\hline Variável & Média & Desvio-padrão & Alpha de Cronbach \\
\hline Confiabilidade & 4,34 & 0,83 & 0,682 \\
Empatia & 4,14 & 0,97 & 0,701 \\
Presteza & 4,07 & 0,98 & 0,718 \\
Segurança & 4,22 & 0,84 & 0,546 \\
Tangibilidade & 3,85 & 1,00 & 0,733 \\
Satisfação & 3,47 & 0,87 & 0,693 \\
\hline Fonte: dados da pesquisa
\end{tabular}

5.3 Impacto do perfil dos usuários nas avaliações dos serviços das UBS

Com o intuito de verificar como o perfil dos usuários interfere na avaliação da qualidade e satisfação dos serviços de saúde oferecidos pelas UBS, procedeu-se a realização do Teste T e do Teste Anova. Desse modo, o Teste T foi calculado em relação ao gênero e o Teste Anova nos demais aspectos do perfil dos usuários (idade, estado civil, escolaridade, renda e UBS).

Segundo Hair Jr. et al. (2005), o Teste T pode ser usado para testar uma hipótese que estabelece que as médias para as variáveis associadas com duas 
ANA CLAUDIA DA ROSA, GABRIELA RORATO GUARIENTI, MATHEUS FROHLICH MARQUETTO, JULIANO PEREIRA RÉGIS GARCIA, ANA LUIZA ROSSATO FACCO, LAÍS VIEIRA TREVISAN, ULISSES ARTHUR CARBONI, JAQUELINE SELINSKE, AMANDA MEDEIROS, PÂMELA RODRIGUES, MARCIA ZAMPIERI GROHMANN, LUCIANA FLORES BATTISTELLA, MARLI MATIKO CAMPOS

amostras ou grupos independentes serão iguais. O Teste T avalia se as diferenças observadas entre as médias de duas amostras ocorreram por acaso ou se houve diferença estatisticamente significativa. Como critério padrão, o nível de significância adotado neste estudo foi de $5 \%($ sig $\leq 0,05)$ e são apresentadas as variáveis que obtiveram diferenças significativas.

Por meio do Teste T, verificou-se que a diferença significativa foi encontrada nos construtos confiança, segurança e satisfação. Na dimensão confiança, os homens obtiveram média 4,52 e desvio-padrão de 0,70, já as mulheres atingiram média 4,25 e desvio-padrão 0,89. Assim, os homens confiam mais do que as mulheres nos serviços prestados pela UBS, e possuem opiniões mais homogêneas nesse construto.

Referente à dimensão confiança, os homens tiveram média 4,34 e desvio-padrão de 0,73 , e as mulheres atingiram média 4,16 e desvio-padrão 0,89. Desse modo, pode-se constatar que os homens atrelam maior importância à segurança dos serviços prestados, bem como apresentam maior concordância nas suas respostas.

Por fim, no construto satisfação verificou-se que os homens novamente atingiram a maior média $(4,17)$ em comparação a média das mulheres $(3,81)$. Além disso, as mulheres obtiveram maior desvio-padrão $(1,09)$, revelando maior discordância entre as respostas.

Portanto, com base nos resultados do Teste T, verificou-se que os homens atingiram as maiores médias nas dimensões confiança, segurança e satisfação, e apresentaram maior homogeneidade nas respostas (menores desvios-padrões). Isso revela que eles confiam mais, têm maior segurança e estão mais satisfeitos do que as mulheres em relação aos serviços prestados pelas UBS. Esses dados são expostos na Tabela 6.

Tabela 6 - Teste T

\begin{tabular}{|l|c|c|c|c|c|c|}
\hline \multirow{2}{*}{ Construto } & \multicolumn{2}{|c|}{ Média } & \multicolumn{2}{c|}{ Desvio-padrão } & \multirow{2}{*}{ Sig } \\
\hline & Fem. & Masc. & Fem. & Masc. & Teste T & \\
\hline Confiança & 4,25 & 4,52 & 0,89 & 0,70 & 11,887 & 0,001 \\
\hline Segurança & 4,16 & 4,34 & 0,89 & 0,73 & 7,577 & 0,006 \\
\hline Satisfação & 3,81 & 4,17 & 1,09 & 0,91 & 8,917 & 0,003 \\
\hline
\end{tabular}

Fonte: dados da pesquisa

Na sequência, procedeu-se a realização do Teste Anova nas demais características do perfil dos entrevistados (idade, estado civil, escolaridade, renda e Unidade de Saúde que o usuário frequenta). Conforme Hair Jr et al. (2005), o Teste Anova consiste em uma análise bivariada, compreende cálculos de frequência e de média, além de cruzar as variáveis.

Desse modo, a idade dos usuários impactou em todas as dimensões avaliadas (Tabela 7). Com base nos resultados, pode-se constatar que os usuários que 
AVALIAÇÃO DOS SERVIÇOS DE SAÚDE:

UMA PESQUISA COM USUÁRIOS DE UNIDADES BÁSICAS VINCULADAS AO PROGRAMA PRÓ-PET SAÚDE

apresentam mais de 70 anos avaliaram de forma mais positiva a empatia (média 4,63) e presteza (média 4,63) dos funcionários das UBS, confiam (média 4,75) mais e tem maior segurança $(4,59)$ com relação aos serviços prestados e estão mais satisfeitos (média 4,44), além disso, esses usuários revelaram maior homogeneidade entre suas respostas. Já os usuários que possuem entre 61 e 70 anos avaliaram de forma mais positiva as instalações físicas (média 4,26) das UBS.

A menor avaliação na dimensão empatia (média 3,87) e tangibilidade $(3,62)$ foi entre os usuários que estão na faixa etária de 21 a 30 anos. Já os usuários que possuem de 31 a 40 anos confiam (média 4,11) menos nos serviços prestados. Além disso, os usuários que têm até 20 anos atrelaram menor avaliação na presteza $(3,65)$ dos funcionários, bem como estão menos satisfeitos (média 3,34) do que os demais usuários. Por fim, os usuários que estão na faixa de 41 a 50 anos tem menos segurança (média 4,08 ) quanto aos serviços prestados pelas UBS.

Tabela 7 - Teste Anova e faixa etária

\begin{tabular}{|c|c|c|c|c|c|}
\hline Construto & Faixa etária & Média & Desvio & $F$ & Sig. \\
\hline Empatia & $\begin{array}{l}\text { Mais de } 70 \text { anos } \\
21 \text { a } 30 \text { anos }\end{array}$ & $\begin{array}{l}4,63 \\
3,87\end{array}$ & $\begin{array}{l}0,51 \\
0,98\end{array}$ & 2,933 & 0,008 \\
\hline Confiabilidade & $\begin{array}{l}\text { Mais de } 70 \text { anos } \\
31 \text { a } 40 \text { anos }\end{array}$ & $\begin{array}{l}4,75 \\
4,11\end{array}$ & $\begin{array}{l}0,40 \\
0,99\end{array}$ & 2,911 & 0,009 \\
\hline Presteza & $\begin{array}{l}\text { Mais de } 70 \text { anos } \\
\text { Até } 20 \text { anos }\end{array}$ & $\begin{array}{l}4,63 \\
3,65\end{array}$ & $\begin{array}{l}0,55 \\
0,97\end{array}$ & 3,853 & 0,001 \\
\hline Segurança & $\begin{array}{l}\text { Mais de } 70 \text { anos } \\
41 \text { a } 50 \text { anos }\end{array}$ & $\begin{array}{l}4,59 \\
4,08\end{array}$ & $\begin{array}{l}0,51 \\
0,90\end{array}$ & 2,496 & 0,022 \\
\hline Tangibilidade & $\begin{array}{l}61 \text { a } 70 \text { anos } \\
21 \text { a } 30 \text { anos }\end{array}$ & $\begin{array}{l}4,26 \\
3,62\end{array}$ & $\begin{array}{l}0,89 \\
1,12\end{array}$ & 2,602 & 0,018 \\
\hline Satisfação & $\begin{array}{l}\text { Mais de } 70 \text { anos } \\
\text { Até } 20 \text { anos }\end{array}$ & $\begin{array}{l}4,44 \\
3,34\end{array}$ & $\begin{array}{l}0,61 \\
1,31\end{array}$ & 4,015 & 0,001 \\
\hline
\end{tabular}

Referente ao estado civil, apenas tangibilidade apresentou diferença estatisticamente significativa dentre as avaliações dos usuários, conforme exposto na Tabela 8. No fator, os divorciados apresentaram as melhores avaliações (média 4,31), já os casados ou em união estável atrelaram as menores avaliações aos equipamentos e instalações das UBS (média 3,72). Desse modo, os usuários divorciados avaliaram de forma mais positiva as instalações e equipamentos das UBS, em comparação aos usuários que são casados ou estão em união estável.

Tabela 8 - Teste Anova e estado civil

\begin{tabular}{c|c|c|c|c|c|}
\hline Construto & UBS & Média & Desvio & F & Sig. \\
\hline Tangibilidade & Divorciado & 4,31 & 0,90 & 3,073 & 0,028 \\
\hline Fonte: dados da pesquisa & Casado/União estável & 3,72 & 0,99 & & \\
\hline
\end{tabular}


ANA CLAUDIA DA ROSA, GABRIELA RORATO GUARIENTI, MATHEUS FROHLICH MARQUETTO, JULIANO PEREIRA RÉGIS GARCIA, ANA LUIZA ROSSATO FACCO, LAÍS VIEIRA TREVISAN, ULISSES ARTHUR CARBONI, JAQUELINE SELINSKE, AMANDA MEDEIROS, PÂMELA RODRIGUES, MARCIA ZAMPIERI GROHMANN, LUCIANA FLORES BATTISTELLA, MARLI MATIKO CAMPOS

Com relação à escolaridade (Tabela 9), foram observadas diferenças estatísticas em quatro dimensões do modelo: empatia, confiabilidade, presteza e tangibilidade. De modo geral, em todos esses fatores, os usuários com maior grau de instrução (superior completo/incompleto) revelaram as menores avaliações, ou seja, apresentaram as menores médias, bem como maior disparidade entre suas respostas. Portanto, esses usuários confiam (média 4,04) menos nos serviços prestados, atrelaram menor avaliação para a empatia (média 3,57 ) e presteza (média 3,63) dos funcionários das UBS e revelaram aspectos físicos das UBS (média 3,58).

Os usuários que não frequentaram a escola revelaram as melhores avaliações quanto a empatia (média 4,32) dos serviços prestados, bem como quanto à estrutura física das UBS (média 4,08). Já os usuários com ensino fundamental completo/incompleto confiam mais nos serviços (média 4,46 ) e acreditam mais na presteza dos serviços prestados pelos profissionais das UBS.

Desse modo, pode-se verificar que usuários com maiores níveis de escolaridade são mais rigorosos nas avaliações dos serviços prestados pelas UBS, ao passo que atribuíram as menores médias as dimensões avaliadas na pesquisa.

Tabela 9 - Teste ANOVA e escolaridade

\begin{tabular}{|c|c|c|c|c|c|}
\hline Construto & Escolaridade & Média & Desvio & $F$ & Sig. \\
\hline Empatia & $\begin{array}{l}\text { Não frequentou a escola } \\
\text { Superior Completo/Incompleto }\end{array}$ & $\begin{array}{l}4,32 \\
3,57\end{array}$ & $\begin{array}{l}0,81 \\
0,97\end{array}$ & 4,703 & 0,003 \\
\hline Confiabilidade & $\begin{array}{l}\text { Ensino Fundamental Completo/Incompleto } \\
\text { Superior Completo/Incompleto }\end{array}$ & $\begin{array}{l}4,46 \\
4,04\end{array}$ & $\begin{array}{l}0,76 \\
0,81\end{array}$ & 3,978 & 0,008 \\
\hline Presteza & $\begin{array}{l}\text { Ensino Fundamental Completo/Incompleto } \\
\text { Superior Completo/Incompleto }\end{array}$ & $\begin{array}{l}4,21 \\
3,63\end{array}$ & $\begin{array}{l}0,91 \\
1,01\end{array}$ & 4,243 &, 006 \\
\hline Tangibilidade & $\begin{array}{l}\text { Não frequentou a escola } \\
\text { Superior Completo/Incompleto }\end{array}$ & $\begin{array}{l}4,08 \\
3,58\end{array}$ & $\begin{array}{l}0,78 \\
0,99\end{array}$ & 5,883 & 0,001 \\
\hline
\end{tabular}

A renda dos usuários causou impacto estatisticamente significativo em três dimensões, sendo elas confiabilidade, presteza e tangibilidade. Desse modo, os usuários que não souberam ou não quiseram revelar a sua renda apresentaram as maiores médias em todas as dimensões impactadas, revelando confiarem (média 4,91) mais nos serviços e na presteza (média 4,80) dos funcionários e avaliaram melhor as instalações físicas $(4,57)$ das UBS. Além disso, esses usuários revelaram menos disparidade entre suas avaliações (menores desvios padrões).

Além disso, os usuários com renda de 3 salários mínimos revelaram menor confiabilidade (média 4,12), e avaliaram de forma menos positiva a presteza no atendimento (média 3,73) e as instalações físicas (média 3,51) das UBS. Os dados referentes ao Teste Anova da renda podem ser visualizados na Tabela 10. 
Tabela 10 - Teste Anova e renda

\begin{tabular}{|c|c|c|c|c|c|}
\hline Construto & Renda & Média & Desvio & F & Sig. \\
\hline Confiabilidade & Não revelou & 4,91 & 0,23 & 2,390 & 0,038 \\
\hline \multirow{2}{*}{ Presteza } & 3 salários mínimos & 4,12 & 1,04 & 2,390 & \\
\hline Não revelou & 4,80 & 0,40 & 2,862 & 0,015 \\
\hline 3 salários mínimos & 3,73 & 1,10 & & \\
\hline Tangibilidade & Não revelou & 4,57 & 0,53 & 4,175 & 0,001 \\
\hline
\end{tabular}

Com relação a UBS, verificou-se que houve diferença significativa em todos os construtos do modelo. Assim, dentre as UBS, na dimensão empatia a CAPS Caminho do Sol apresentou melhor avaliação (maior média: 4,77) e menor desvio-padrão $(0,40)$ e a UBS Alto da Boa Vista teve a menor avaliação (menor média: 3,45$)$ e o maior desvio $(1,06)$.

Em confiabilidade, a UBS Lídia teve uma melhor avaliação (média 4,90) e maior homogeneidade nas respostas (desvio 0,30), e novamente a unidade Alto da Boa Vista obteve a avaliação mais baixa (menor média: 3,76 ) e maior discordância nas respostas (maior desvio padrão: 0,94).

No fator presteza, a unidade da Lídia atingiu novamente a melhor avaliação na opinião dos usuários (maior média: 4,67 ) e revelou maior concordância entre as opiniões (menor desvio: 0,53 ). Já a UBS da Urlândia revelou a menor avaliação (menor média: 3,35) e menor concordância nas opiniões dos usuários pesquisados (maior desvio: 1,14).

Sobre a segurança, a UBS Lídia revelou novamente a melhor avaliação (maior média: 4,75 ) e respostas mais homogêneas dentre os entrevistados (menor desvio: 0,51) e a unidade do Alto da Boa Vista atingiu a menor média $(3,72)$ e maior discordância entre as respostas dos usuários (maior desvio: 0,82).

Em tangibilidade, a maior média $(4,62)$ e menor discordância entre as respostas (menor desvio: 0,64 ) também foi encontrada na unidade da Lídia, e a unidade da Urlândia obteve a menor média $(3,24)$, bem como maior disparidade entre as opiniões dos usuários (maior desvio: 0,91 ).

No fator satisfação a melhor avaliação (média 4,53) e menor disparidade nas respostas (desvio 0,48 ) pode ser observada na CAPS Caminho do Sol, e a menor avaliação e maior discordância entre os usuários foi verificada na UBS Urlândia (média 2,89).

Desse modo, verifica-se que dentre os fatores do modelo, os usuários da UBS Lídia confiam, tem maior segurança e acreditam na presteza dos serviços prestados pelos funcionários das UBS, avaliaram melhor os aspectos físicos da UBS e estão mais satisfeitos. Já os usuários do Alto da Boa Vista revelaram menor empatia por parte dos funcionários dessa UBS, confiam menos e tem menos segurança nos serviços que são prestados. Os usuários da CAPS Caminho do Sol avaliaram de melhor forma a empatia dos funcionários que trabalham nessa UBS e estão mais satisfeitos com os serviços. Além disso, os usuários da 
ANA CLAUDIA DA ROSA, GABRIELA RORATO GUARIENTI, MATHEUS FROHLICH MARQUETTO, JULIANO PEREIRA RÉGIS GARCIA, ANA LUIZA ROSSATO FACCO, LAÍS VIEIRA TREVISAN, ULISSES ARTHUR CARBONI, JAQUELINE SELINSKE, AMANDA MEDEIROS, PÂMELA RODRIGUES, MARCIA ZAMPIERI GROHMANN, LUCIANA FLORES BATTISTELLA, MARLI MATIKO CAMPOS

Urlândia revelaram menor presteza dentre os serviços prestados, problemas com aspectos físicos dessa UBS e estão menos satisfeitos com os serviços prestados. Os resultados do Teste Anova podem ser verificados na Tabela 11.

\begin{tabular}{|c|c|c|c|c|c|}
\hline Construto & UBS & Média & Desvio & $F$ & Sig. \\
\hline Empatia & $\begin{array}{l}\text { CAPS Caminho do Sol } \\
\text { Alto da Boa Vista }\end{array}$ & $\begin{array}{l}4,77 \\
3,45\end{array}$ & $\begin{array}{l}0,40 \\
1,06\end{array}$ & 8,180 & 0,000 \\
\hline Confiabilidade & $\begin{array}{c}\text { Lídia } \\
\text { Alto da Boa Vista }\end{array}$ & $\begin{array}{l}4,90 \\
3,76\end{array}$ & $\begin{array}{l}0,30 \\
0,94\end{array}$ & 7,383 & 0,000 \\
\hline Presteza & $\begin{array}{l}\text { Lídia } \\
\text { Urlândia }\end{array}$ & $\begin{array}{l}4,67 \\
3,35\end{array}$ & $\begin{array}{l}0,53 \\
1,14\end{array}$ & 9,451 & 0,000 \\
\hline Segurança & $\begin{array}{c}\text { Lídia } \\
\text { Alto da Boa Vista }\end{array}$ & $\begin{array}{l}4,75 \\
3,72\end{array}$ & $\begin{array}{l}0,51 \\
0,82\end{array}$ & 5,587 & 0,000 \\
\hline Tangibilidade & $\begin{array}{l}\text { Lídia } \\
\text { Urlândia }\end{array}$ & $\begin{array}{l}4,62 \\
3,24\end{array}$ & $\begin{array}{l}0,64 \\
0,91\end{array}$ & 8,299 & 0,000 \\
\hline Satisfação & $\begin{array}{l}\text { CAPS Caminho do Sol } \\
\text { Urlândia }\end{array}$ & $\begin{array}{l}4,53 \\
2,89\end{array}$ & $\begin{array}{l}0,48 \\
1,24\end{array}$ & 13,993 & 0,000 \\
\hline
\end{tabular}

Portanto, com base nos resultados do Teste T e Teste Anova, pôde-se verificar as influências do perfil dos usuários na avaliação da qualidade e satisfação dos serviços de saúde. Assim, a idade e a UBS de atendimento foram as características do perfil dos usuários que mais impactaram as avaliações dos serviços, ambos impactando em todos os construtos do modelo (100,00\%). Referente à idade, verificou-se que os usuários que apresentam as idades mais elevadas (entre 61 até 70 e acima de 70 anos) avaliaram de forma mais positiva a qualidade e a satisfação dos serviços prestados pelas UBS. Já as menores avaliações da qualidade e satisfação dos serviços são dos usuários que estão nas faixas etárias entre 20 e 50 anos.

Quanto a UBS, as maiores avaliações sobre a empatia e satisfação foram dos usuários da CAPS Caminho do Sol e as melhores avaliações referentes a confiabilidade, presteza, segurança e tangibilidade foram encontradas nos usuários da Lídia. Dessa forma, essas UBS foram as melhores avaliadas quanto à qualidade e satisfação dos serviços de saúde. Já as menores avaliações sobre a presteza dos funcionários, aspectos físicos da UBS e satisfação dos serviços foram observadas na UBS Urlândia. Do mesmo modo, as menores avaliações quanto à empatia dos funcionários, confiabilidade e segurança dos serviços foram encontradas na UBS do Alto da Boa Vista. Assim, as UBS Urlândia e Alto da Boa Vista revelaram as menores avaliações quanto a qualidade e satisfação dos serviços de saúde prestados à população.

Na sequência, o nível de escolaridade gerou diferenças estatisticamente significativas em quatro construtos, sendo eles empatia, confiabilidade, presteza e tangibilidade, representando $66,67 \%$ dos fatores. Pode-se verificar que os usuários com os maiores níveis de escolaridade são mais críticos quanto à 
AVALIAÇ̃̃O DOS SERVIÇOS DE SAÚDE:

avaliação dos serviços, ao passo que atrelaram as menores avaliações as dimensões empatia, confiabilidade, presteza e tangibilidade.

O gênero e a renda causaram impacto em 3 dimensões (50,00\%), sendo que o gênero impactou nos construtos confiança, segurança e satisfação, e renda impactou nos fatores confiabilidade, presteza e tangibilidade. Quanto ao gênero, os homens revelaram maior confiança, segurança e satisfação quanto aos serviços prestados pelas UBS. Referente à renda, os usuários que não souberam ou não quiseram revelar a sua renda avaliaram de forma mais positiva a confiança, a presteza dos funcionários e as instalações físicas das UBS, já os usuários com renda de três salários mínimos revelaram menor confiabilidade e avaliaram de forma menos positiva a presteza no atendimento e as instalações físicas das UBS.

Por fim, o estado civil impactou apenas a dimensão tangibilidade (33,33\%), em que os usuários divorciados avaliaram de forma mais positiva as instalações e equipamentos das UBS, em comparação aos usuários que são casados ou estão em união estável.

Supondo que as características tivessem impactado em todas as dimensões, as características teriam impactado em $100 \%$ do modelo, contudo, verificou-se que ao total, as características impactaram em 23, representando 63,89\%, ou seja, gerando um impacto significativo na avaliação dos usuários sobre os serviços prestados pelas UBS. Esses resultados são apresentados no Quadro 3.

\begin{tabular}{|c|c|c|c|}
\hline Característica & Construtos & Total & Percentual \\
\hline Gênero & Confiança, segurança e satisfação & 03 & $50,00 \%$ \\
\hline Idade & $\begin{array}{c}\text { Empatia, confiabilidade, presteza, segurança, tangibilidade e } \\
\text { satisfação }\end{array}$ & 06 & $100,00 \%$ \\
\hline Estado civil & Tangibilidade & 01 & $33,33 \%$ \\
\hline Escolaridade & Empatia, confiabilidade, presteza e tangibilidade & 04 & $66,67 \%$ \\
\hline Renda & Confiabilidade, presteza, tangibilidade & 03 & $50,00 \%$ \\
\hline UBS & $\begin{array}{c}\text { Empatia, confiabilidade, presteza, segurança, tangibilidade e } \\
\text { satisfação }\end{array}$ & 06 & $100,00 \%$ \\
\hline TOTAL & $\mathbf{2 3}$ & \multicolumn{2}{|l}{} \\
\hline Percentual & $\mathbf{6 3 , 8 9 \%}$ & & \\
\hline
\end{tabular}

Quadro 3 - Resumo das influências do perfil nas dimensões dos modelos teóricos

Fonte: dados da pesquisa

\section{Considerações finais}

O PET-Saúde é uma estratégia associada ao PRÓ-Saúde que preconiza ações para a transformação da formação profissional em saúde, mediante maior integração entre ensino, serviço e comunidade. Dessa forma, a presente pesquisa objetivou mensurar a qualidade e a satisfação dos serviços prestados e verificar como o perfil dos usuários interfere na avaliação dessas dimensões. Assim sendo, realizou-se um estudo quantitativo e descritivo com 373 usuários de dez UBS de Santa Maria/RS, por meio da adaptação da Escala SERVQUAL proposta por Parasuraman, Zeithaml e Berry (1998) e da Escala de Satisfação de Oliver (1997). 
ANA CLAUDIA DA ROSA, GABRIELA RORATO GUARIENTI, MATHEUS FROHLICH MARQUETTO, JULIANO PEREIRA RÉGIS GARCIA, ANA LUIZA ROSSATO FACCO, LAÍS VIEIRA TREVISAN, ULISSES ARTHUR CARBONI, JAQUELINE SELINSKE, AMANDA MEDEIROS, PÂMELA RODRIGUES, MARCIA ZAMPIERI GROHMANN, LUCIANA FLORES BATTISTELLA, MARLI MATIKO CAMPOS

Com base nos resultados, constatou-se que o perfil dos usuários que compuseram a amostra foi em sua maioria feminino, na faixa etária de 41 a 50 anos, estado civil casado/união estável, renda de um salário mínimo, e nível de escolaridade fundamental completo/incompleto. Assim sendo, o perfil predominante dos usuários participantes da atual pesquisa foi de uma população adulta, do sexo feminino e de baixa escolaridade.

Por meio da análise das médias e desvios-padrões dentre cada UBS pesquisada identificou-se que de modo geral as dez UBS foram bem avaliadas. Os usuários pesquisados sentem confiança e segurança nos serviços prestados pelas UBS, afirmam que os profissionais da saúde possuem conhecimento e empatia no atendimento, e atrelam as menores avaliações às condições dos equipamentos e as instalações das UBS, bem como a satisfação geral com relação aos serviços prestados nas UBS.

Com relação ao impacto do perfil dos usuários, verificou-se que esse perfil interfere significativamente na avaliação dos serviços prestados pelas UBS. Assim, a idade e a UBS de atendimento foram as características que mais impactaram as avaliações dos serviços, seguidos do nível de escolaridade, gênero e a renda, sendo que a característica do perfil que menos interferiu na avaliação da qualidade e satisfação dos serviços prestados refere-se ao estado civil.

Com relação às limitações, cita-se que alguns usuários possuíam receio de fornecer informações, além disso, apesar da revisão do instrumento de coleta de dados, verificou-se que durante a aplicação dos questionários, alguns pesquisados não compreenderam algumas questões. Ressalta-se que houve dificuldade na obtenção dos dados, visto que os questionários eram aplicados em horário de funcionamento das UBS.

A partir deste trabalho, verificou-se a necessidade em se realizar um estudo exploratório e qualitativo, para extrair dados mais precisos com relação aos entrevistados, traçando novos pontos que tem que ser melhorados nas UBS, pois ao questionar os usuários, os mesmos relataram aos pesquisadores outros aspectos que devem ser melhorados nas UBS, sendo um dos principais aspectos relatados a redução das filas para agendamento de consultas. Além disso, sugere-se a aplicação da pesquisa em outras cidades, a fim de traçar as avaliações dos usuários, servindo de base para o aprimoramento da oferta dos serviços de saúde. Dessa forma, os resultados encontrados na pesquisa podem servir de base para a constante melhoria dos serviços públicos de saúde oferecidos pelas UBS atuantes no PRÓ-PET-Saúde em Santa Maria/RS, pois revelou a avaliação dos usuários sobre diversos aspectos no atendimento. Assim sendo, a pesquisa pode dar suporte a novos estudos realizados para mensurar a opinião dos usuários dos serviços de saúde no Brasil, cuja avaliação é muito importante para o constante aprimoramento dos serviços prestados. 
AVALIAÇ̃̃O DOS SERVIÇOS DE SAÚDE:

UMA PESQUISA COM USUÁRIOS DE UNIDADES BÁSICAS VINCULADAS AO PROGRAMA PRÓ-PET SAÚDE

\section{Referências}

AGUILAR-DA-SILVA, R. H., Scapin, L. T., \& Batista, N. A. Avaliação da formação interprofissional no ensino superior em saúde: aspectos da colaboração e do trabalho em equipe. Avaliação (Campinas), v. 16, n. 1, p. 16784, 2011.

ARAÚJO, F. C. et al. O aprender e o orientar na atenção primária: relato de experiência de um semestre de atividades no PET-Saúde. Rev. bras. educ. med., v. 36, n. 1, p. 164-168, 2012

AYRES, J. R. C. M. Cuidado e reconstrução das práticas de saúde. Interface-Comunicação, Saúde, Educação, v. 8, n. 14, p. 73-91, 2004.

BRASIL. Ministério da Saúde. Secretaria de Atenção à Saúde. Avaliação na Atenção Básica em Saúde: caminhos da institucionalização. Coordenação técnica: Instituto de Saúde Coletiva da Universidade Federal da Bahia e Instituto Materno Infantil Prof. Fernando Figueira (IMIP). Brasília, DF: Ministério da Saúde, 2005.

CEZARIO, S. M. et al. Association of high-density lipoprotein and apolipoprotein e genetic variants with age-related macular degeneration. Arq Bras Oftalmol, v. 78, n. 2, p. 85-8, 2015.

DALAZANA, F., Ferreira, G. M. V., \& Talamini, E. 2007. Qualidade na percepção dos clientes internos: uma aplicação da escala SERVQUAL em uma indústria metalúrgica do Rio Grande do Sul. In: SIMPÓSIO DE EXCELÊNCIA EM GESTÃO E TECNOLOGIA, 4, Resende, 2007. Anais... Resende, AEDB.

DONABEDIAN, A. Continuity and Change in the Quest for Quality. Clinical performance and quality health care, v. 1, n. 1, p. 9-16, 1993.

ESPERIDIÃO, M., \& Trad, L. A. B. Avaliação de satisfação de usuários. Ciência e saúde coletiva, v.10, p. 303-312, 2005.

FERREIRA, V. S. et al. PET-Saúde: uma Experiência Prática de Integração Ensino-serviço-comunidade. Revista brasileira de educação médica, v. 36, n. 1, p. 147-151, 2012.

GOUVEIA, G. C. Avaliação da satisfação dos usuários com o Sistema de Saúde brasileiro. Recife, 2009. Tese (Doutorado em Saúde Pública) - Centro de Pesquisas Aggeu Magalhães, FIOCRUZ.

HAIR JR., Joseph F. et al. Análise Multivariada de Dados. 6. ed. Porto Alegre: Bookman, 2005.

INSTITUTO BRASILEIRO DE GEOGRAFIA E ESTATí́sTICA [IBGE]. Cidades, Santa Maria/RS. Disponível em: http:// cidades.ibge.gov.br/xtras/perfil.php?codmun=431690. Acesso em 12.03.2017.

LEMME, A. C., Noronha, G., \& Resende, J. B. A satisfação do usuário em hospital universitário. Revista de saúde pública, São Paulo, v. 25, n. 1, p. 41-46, 1991.

MEDINA, Maria Guadalupe et al. Uso dos modelos teóricos na avaliação em saúde: aspectos conceituais e operacionais. In: HARTZ, Zulmira Maria de Araújo; VIEIRA-DA-SILVA, Ligia Maria (Org). Avaliação em saúde: dos modelos teóricos à prática na avaliação em programas e sistemas de saúde. Rio de Janeiro: Ed. Fiocruz / Salvador: EDUFBA, 2005. p. 41-63.

MENDES, A. C. G et al. Avaliação da satisfação dos usuários com a qualidade do atendimento nas grandes emergências do Recife, Pernambuco, Brasil. Revista Brasileira de Saúde Materno Infantil, v. 9, n. 2, p. 157-165, 2009.

MINISTÉRIO DA SAÚDE. Portal da Saúde. Disponível em: http://portalsaude.saude.gov.br/index.php/cidadao/ entenda-o-sus. Acesso em 21.02.2017.

MINISTÉRIO DO PLANEJAMENTO. Unidade Báscia de Saúde. Disponível em: http://www.pac.gov.br/infraestruturasocial-e-urbana/ubs-unidade-basica-de-saude/rs. Acesso em 21.02.2017.

MORAIS, F. R. R. et al. A importância do PET-Saúde para a formação acadêmica do enfermeiro. Trab. educ. Saúde, v. 10, n. 3, 2012.

OLIVER, Richard L. Satisfaction: A behavioral perspective on the consumer. Boston: McGraw Hill, 1997.

PARASURAMAN, A., Zheitmal, V. A., \& Berry, L. L. SERVQUAL: a multipleitem scale for measuring consumer perceptions of service quality. J. Retailing. v. 64, n. 41, 1998.

PENA, M. et al. The use of the quality model of Parasuraman, Zeithaml and Berry in health services. Revista da Escola de Enfermagem da USP, v. 45, n. 5, 2013. 
ANA CLAUDIA DA ROSA, GABRIELA RORATO GUARIENTI, MATHEUS FROHLICH MARQUETTO, JULIANO PEREIRA RÉGIS GARCIA, ANA LUIZA ROSSATO FACCO, LAÍS VIEIRA TREVISAN, ULISSES ARTHUR CARBONI, JAQUELINE SELINSKE, AMANDA MEDEIROS, PÂMELA RODRIGUES, MARCIA ZAMPIERI GROHMANN, LUCIANA FLORES BATTISTELLA, MARLI MATIKO CAMPOS

PEREIRA, J. G. Articulação ensino-serviço para a construção do modelo da vigilância da saúde: em foco o Distrito do Butantã. São Paulo, 2007. Dissertação (Mestrado em Enfermagem), Universidade de São Paulo.

PESTANA, Maria Helena, \& GAGEIRO, João Nunes. Análise de dados para ciências sociais - a complementaridade do SPSS. 4. ed. Lisboa : Edições Lisboa, 2005.

RAMOS, D. D, \& Lima, M. A. D. S. Acesso e acolhimento aos usuários em uma unidade de saúde de Porto Alegre, Rio Grande do Sul, Brasil. Cadernos de saúde pública, v. 1, n. 19, p.27-34, 2003.

RIBEIRO, D. F. Qualidade em serviços públicos de saúde: a percepção dos usuários do hospital universitário em um município paraibano. Rio de Janeiro, 2010. Dissertação (Mestrado Profissional em Saúde Pública), Fiocruz.

RIGHI, A. W., Schmidt, A. S., \& Venturini, J. C. Qualidade em serviços públicos de saúde: uma avaliação da estratégia saúde da família. Revista Produção Online, v. 10, n. 3, p. 649-669, 2010.

SILVA, A. A. F. A percepção da qualidade do serviço e a satisfação dos usuários das Unidades Básicas de Saúde de Caraguatatuba. Caraguatatuba, 2016. Trabalho de Conclusão de Curso (Tecnologia em processos gerenciais), Instituto Federal de São Paulo.

SILVA, L. M. V., \& Formigli, V. L. A. Avaliação em Saúde: limites e perspectivas. Cadernos de saúde pública, v. 10, n. 1, p. 80-91, 1994

TANAKA, Oswaldo Yoshimi; MELO, C. Reflexões sobre a avaliação em serviços de saúde e a adoção das abordagens qualitativa e quantitativa. In: BOSI, Maria Lúcia Magalhães; MERCADO, Francisco Javier (Org). Pesquisa qualitativa de serviços de saúde. Petrópolis: Vozes, 2004. p. 121- 136.

TRAVASSOS, C., \& Martins, M. Uma revisão sobre os conceitos de acesso e utilização de serviços de saúde. Cadernos de saúde pública, v. 20, p. 190-198, 2004.

VAITSMAN, J, \& Andrade, G. R. B. Satisfação e responsividade: formas de medir a qualidade e a humanização da assistência à saúde. Ciência e saúde coletiva, v.10, n. 3, p.599-613, 2005.

VIEIRA-DA-SILVA, Ligia Maria. Conceitos, abordagens e estratégias para a avaliação em saúde In: HARTZ, Zulmira Maria de Araújo; VIEIRA-DA-SILVA, Ligia Maria (Org). Avaliação em saúde: Dos modelos teóricos à prática na avaliação de programas e sistemas de saúde. Salvador: EDUFBA; Rio de Janeiro: FIOCRUZ, 2005. p. 15-39.

WEISS, L. G. Patient satisfaction with primary medical care: evaluation of sociodemographic and predispositional factors. Medical Care, v. 26, p.383-392, 1988. 
